\title{
PML-Nuclear Bodies Regulate the Stability of the Fusion Protein Dendra2-Nrf2 in the Nucleus
}

\author{
Andrea Flores Burroughs ${ }^{a}$ Sylvia Eluhu ${ }^{b}$ Diva Whalen ${ }^{b} \quad J . S h a w n$ Goodwin ${ }^{b}$ \\ Amos M. Sakwe ${ }^{b}$ Ifeanyi J. Arinze ${ }^{c}$ \\ aDepartment of Neuroscience and Pharmacology, ${ }^{b}$ Department of Biochemistry and Cancer Biology, \\ School of Graduate Studies and Research, 'Department of Physiology, School of Medicine, Meharry \\ Medical College, Nashville, TN, USA
}

\section{Key Words}

Dendra2 - Fluorescent protein • Nrf2 • Nuclear degradation • Proteasome • Photoconversion - Cell biology • Promyelocytic leukemia-nuclear bodies • PyFDAP

\begin{abstract}
Background/Aims: Nuclear factor erythroid 2-related factor 2 (Nrf2) is a basic leucine-zipper transcription factor essential for cellular responses to oxidative stress. Degradation of Nrf2 in the cytoplasm, mediated by Keap1-Cullin3/RING box1 (Cul3-Rbx1) E3 ubiquitin ligase and the proteasome, is considered the primary pathway controlling the cellular abundance of Nrf2. Although the nucleus has been implicated in the degradation of Nrf2, little information is available on how this compartment participates in degrading Nrf2. Methods: Here, we fused the photoconvertible fluorescent protein Dendra2 to Nrf2 and capitalized on the irreversible change in color (green to red) that occurs when Dendra2 undergoes photoconversion to study degradation of Dendra2-Nrf2 in single live cells. Results: Using this approach, we show that the half-life $\left(t_{1 / 2}\right)$ of Dendra2-Nrf2 in the whole cell, under homeostatic conditions, is $35 \mathrm{~min}$. Inhibition of the proteasome with MG-132 or induction of oxidative stress with tert-butylhydroquinone ( $t \mathrm{BHQ}$ ) extended the half-life of Dendra2-Nrf2 by 6 - and 28-fold, respectively. By inhibiting nuclear export using Leptomycin $B$, we provide direct evidence that degradation of Nrf2 also occurs in the nucleus and involves PML-NBs (Promyelocytic Leukemia-nuclear bodies). We further demonstrate that co-expression of Dendra2-Nrf2 and Crimson-PML-I lacking two PML-I sumoylation sites (K65R and K490R) changed the decay rate of Dendra2-Nrf2 in the nucleus and stabilized the nuclear derived Nrf2 levels in whole cells. Conclusion: Altogether, our findings provide direct evidence for degradation of Nrf2 in the nucleus and suggest that modification of Nrf2 in PML nuclear bodies contributes to its degradation in intact cells.
\end{abstract}




\section{Introduction}

Nuclear factor erythroid 2-related factor 2 (Nrf2) is a basic leucine-zipper transcription factor that is essential for cellular response to oxidative stress [1-3]. When activated, it binds to the antioxidant response element (ARE) within target gene promoters to regulate basal and inducible expression of hundreds of genes that harbor this element in their promoters $[1,2,4]$. Such genes include those that code for proteins that function in DNA repair processes, the ubiquitin-26S proteasome required for protein degradation, cellular signaling, gene transcription (as transcription factors), chaperoning/heat shock functions, protein trafficking, CNS-specific functions, cellular adhesion, and various aspects of intermediary metabolism [4-8].

Decreasing the availability of Nrf2 through degradation is regarded as the primary means of regulating its cellular abundance, and thereby its function. To date, this degradation is understood to be mediated primarily by the Keap1-Cul3-Rbx1 complex which ubiquitylates Nrf2 in the cytoplasm, followed by proteasomal degradation of the ubiquitylated Nrf2 $[2,9-11]$. It is becoming increasingly apparent that Nrf2 can also be degraded via Keap1independent mechanism(s) [12-14]. Moreover, it appears that Nrf2 can also be degraded in the nucleus $[9,14-17]$, but little is known about the mechanism(s) and dynamics of this nuclear degradation.

The availability of photoconvertible fluorescent proteins (PCFPs) provide an excellent tool for directly tracking/monitoring the degradation of an individual protein in living cells $[18,19]$. With light of specific wavelength and intensity, PCFPs irreversibly change color upon photoconversion [20]. By fusing a PCFP to a candidate protein, it is possible to take advantage of this change in color to track the movement and/or degradation of the candidate protein within cells $[19,21]$. The PCFP Dendra2 is green when expressed in cells and changes color irreversibly to red when a laser is applied at 405 or $488 \mathrm{~nm}$ [20].

Until now, directly monitoring degradation of Nrf2 in living cells has not been attempted. Here, we fused Dendra2 to Nrf2 and capitalized on the photoconversion property to investigate degradation of Dendra2-Nrf2 in single live cells via confocal microscopy. Decay of red fluorescence of photoconverted Dendra2-Nrf2 was monitored in real time as a read-out of degradation of Nrf2 in the whole cell and within the nuclear compartment. Using photoconversion of Dendra2 and PyFDAP (Python Fluorescence Decay After Photoconversion) analysis software, this study provides direct evidence that Nrf2 is degraded in the nucleus and underscores the importance of PML-NBs in the regulation of Nrf2-mediated response to oxidative stress.

\section{Materials and Methods}

\section{Plasmids}

Plasmids expressing the fusion proteins Dendra2-Nrf2 and Crimson-PML-I were constructed using In-fusion HD EcoDry Cloning kit (Clontech Laboratories Inc., Mountain View, CA). Briefly, mouse Nrf2 or human PML-I genes were PCR amplified from their templates (for all online suppl. material, see www. karger.com/doi/ 10.1159/000490033, Suppl. Table S1) and cloned in frame with Dendra2 or Crimson in their respective vectors. The purified PCR products and linearized vectors were incubated with In-fusion HD EcoDry reaction mixture according to the manufacture. We also generated a Crimson-PML-I construct in which two PML-I sumoylation sites were mutated (K65R and K490R), herein designated Crimson-PMLI-2KR mutant (Vector Builder, Santa Clara, CA). The resulting Dendra2-Nrf2 and Crimson-PML-I constructs were verified by restriction enzyme digestion and by DNA sequencing.

Cell culture and chemicals

HepG2 cells were obtained from Sigma-Aldrich (St. Louis, MO) and cultured in minimum essential medium (MEM) supplemented with 10\% fetal bovine serum (FBS), 2 mM L-glutamine, 1 mM sodium pyruvate, 1X MEM nonessential amino acids (Invitrogen), and antibiotics (100 units of penicillin and 100 


\section{Cellular Physiology Cell Physiol Biochem 2018;47:800-816

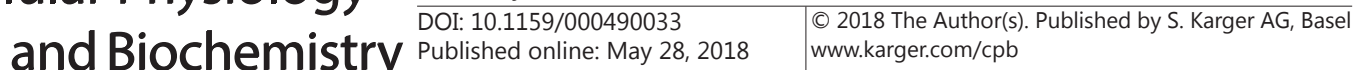

Burroughs et al.: PML-Nuclear Bodies Regulate the Stability of Nrf2

$\mu$ g of streptomycin per $\mathrm{ml}$ ) at $37{ }^{\circ} \mathrm{C}$ in $95 \%$ air, $5 \% \mathrm{CO}_{2}$ atmosphere, as previously described [22]. Mirus TransIT-X2 transfection reagent was purchased from Mirus Bio (Madison, WI) MIR6000. The following items were purchased from Sigma (St. Louis, MO): Dimethyl Sulphoxide (DMSO) Hybri-Max, D2650; tertbutylhydroquinone ( $t$ BHQ), 112941 and Leptomycin B, L2913-5UG. MG-132, BML-P1102-0025; NFKappaB SN50, BML-P600-005 and NF-KappaB SN50M (Mutant), BML-P601-005 were purchased from Enzo Life Sciences (Farmingdale, NY). Geneticin, 11811-031 and Hoechst 33342, R37605 were purchased from Life Technologies (Grand Island, NY).

\section{Western blot analysis}

Whole cell lysates were prepared by lysing cells in non-denaturing lysis buffer [Tris-HCl pH 7.5 (20 $\mathrm{mM}), \mathrm{NaCl}(150 \mathrm{mM})$, EDTA $(1 \mathrm{mM})$, Triton X-100 (1\%), sodium orthovanadate (1 mM), DTT (5 mM), PMSF $(1 \mathrm{mM})$, Protease Inhibitor Cocktail $(10 \mu \mathrm{M} / \mathrm{ml})]$. Equal amounts of protein were separated in 8\% SDS gels, transferred to nitrocellulose membranes and immunoblotted using antibodies against the following proteins purchased from Santa Cruz Biotechnology (Santa Cruz, CA): Nrf2 (sc-13032 Lot\# 51413) and PML (sc-5621 Lot\# C2911). Dendra2 (TA180094 Lot\# 1013) and Crimson (TA183017 Lot\# 0114) were purchased from Origene Tech. Inc., (Rockville, MD). For densitometric analysis, HO-1 protein was quantified using UN-SCAN-IT software (Silk Scientific, Inc., Orem, UT). The ratio of HO-1 protein to LDH loading control was calculated using the densitometry values. The fold change of HO-1 protein in the presence of $t \mathrm{BHQ}$ was normalized to the DMSO control.

\section{Stable transfection of HepG2 cells expressing Dendra2 or Dendra2-Nrf2}

HepG2 cells $\left(5 \times 10^{5}\right)$ were seeded in a 6-well plate and transiently transfected with pDendra2 for $48 \mathrm{~h}$. The cells were then trypsinized and collected in 1X Hank's Balanced Salt Solution (Life Technologies, Grand Island, NY) supplemented with calcium chloride ( $1 \mathrm{mM})$, magnesium chloride $(0.5 \mathrm{mM})$ and FBS (0.5\%). The cells were subsequently sorted using a BD FACSAria III platform (Vanderbilt University flow cytometry core facility, Nashville, TN) and collected in $2 \mathrm{ml}$ of complete MEM. Two weeks later, the cells were sorted again and grown to confluency in a T-25 flask. HepG2 cells that stably expressed Dendra2 were then harvested and stored in liquid nitrogen. For stable transfection of Dendra2-Nrf2, HepG2 cells $\left(5 \times 10^{5}\right)$ were seeded in a 6-well plate and transiently transfected with pDendra2-Nrf2. Forty-eight hours later, the cells were treated with Geneticin $(200 \mu \mathrm{g} / \mathrm{ml})$ for 12 days. The concentration of Geneticin was then increased to $500 \mu \mathrm{g} / \mathrm{ml}$ for 18 days and subsequently reduced to $300 \mu \mathrm{g} / \mathrm{ml}$ for the remainder of the selection process. HepG2 cells that stably expressed Dendra2-Nrf2 were then harvested and stored in liquid nitrogen.

\section{Immunofluorescence to detect PML protein}

HepG2 cells $\left(5 \times 10^{5}\right)$ were seeded onto poly-D-lysine-coated coverslips in 6-well plates in $2 \mathrm{ml}$ of medium and incubated overnight at $37{ }^{\circ} \mathrm{C}$. Some cells were then transfected with expression plasmid(s) indicated in the appropriate figures, using Mirus TransIT-X2 transfection reagent. Twenty-four hours after transfection, the cells were harvested by removing the medium, and rinsing once with $1 \mathrm{ml}$ of $1 \mathrm{X}$ phosphate-buffered saline (PBS). The cells were fixed with $500 \mu \mathrm{l}$ of $3.7 \%$ formaldehyde solution for 10 $\mathrm{min}$ at room temperature, then washed twice with $1 \mathrm{ml}$ of $1 \mathrm{X}$ PBS followed by a 5 -min wash with $1 \mathrm{ml}$ of 0.1 M glycine-Tris buffer ( $\mathrm{pH}$ 7.4), and rinsed with $1 \mathrm{ml}$ of $1 \times$ PBS. Next, the cells were incubated in blocking solution (1\% bovine serum albumin in PBS containing 1\% Triton X-100) for $30 \mathrm{~min}$ at room temperature. After removing the blocking solution, the cells were incubated with anti-PML antibody (sc-966, Santa Cruz Biotechnology, Santa Cruz, CA) diluted 1:50 in blocking solution for $1 \mathrm{~h}$ at room temperature. The primary antibody was removed by aspiration, and the coverslips were washed three times with PBS (5 min for each wash). Coverslips were then incubated with Alexa Fluor633 goat anti-mouse IgG (Invitrogen) diluted 1:2, 500 in blocking solution for $1 \mathrm{~h}$ at room temperature in the dark and then washed and stained for nuclei using a drop of Prolong Gold Antifade with DAPI (Invitrogen) and dried overnight at $4{ }^{\circ} \mathrm{C}$ for visualization. PML protein was visualized on a Nikon A1R confocal laser scanning microscope at excitation/emission wavelengths of 640/662-737 nm, respectively.

Photoconversion of Dendra2 in live cells

HepG2 cells $\left(5 \times 10^{5}\right)$ were seeded on glass bottom MatTek dishes then transfected with pDendra2, pEGFP-Nrf2 or pDendra2-Nrf2, and visualized $48 \mathrm{~h}$ later using a Nikon A1R confocal microscope. 


\section{Cellular Physiology Cell Physiol Biochem 2018;47:800-816

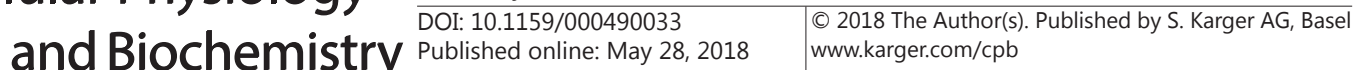

Burroughs et al.: PML-Nuclear Bodies Regulate the Stability of Nrf2

Photoconversion was achieved by illuminating the region of interest using a laser at $405 \mathrm{~nm}$ wavelength with a power of $100 \mathrm{~mW}$ at $100 \%$ for $2 \mathrm{sec}$ (whole cell) or $0.5 \mathrm{sec}$ (nucleus). Using a resonant scanner, images were captured in the green (488/505-550 nm) and red (561/570-620 nm) channels every 0.06 seconds. In other experiments a galvanometer scanner was used to capture images every 45 seconds.

\section{Cell viability assay using fluorescent microscopy analysis}

Non-transfected HepG2 cells $\left(5 \times 10^{5}\right)$ were plated in glass bottom MatTek dishes and visualized 48 $\mathrm{h}$ later. Some cells were demarcated around the cells margin and a $405 \mathrm{~nm}$ laser pulse was applied for 2.0 sec to the indicated area, to simulate photoconversion. After $3 \mathrm{~h}$ of imaging, using the same experimental settings on the microscope, the cells were labeled with $5 \mathrm{X}$ concentration of Live and Dead Cell assay reagent (Abcam- Cat. \# ab115347, Lot \# GR313559) for 10 minutes and quantitation of cell viability was carried out by fluorescent microscopy. Images were captured in the green (494/515 nm) and red (528/617 nm) channels before and after application of the laser.

\section{Real Time PCR of heme oxygenase-1 in cells expressing Dendra2-Nrf2}

HepG2 cells $\left(5 \times 10^{5}\right)$ were transfected with $2.5 \mu \mathrm{g}$ of pDendra2 or pDendra2-Nrf2 and grown for 48 h. Some cells were treated with $t$ BHQ $(20 \mu \mathrm{M})$ for $8 \mathrm{~h}$ prior to harvesting the cells. The RNA was purified using the Pure Link RNA mini kit (Thermo Fisher Cat. \# 12183018A) and synthesis of first strand cDNA was generated using iScript cDNA synthesis kit (Bio-Rad, Cat. \# 1708891). Real time PCR was performed using gene specific Taqman primers for HO-1 and $\beta$ - actin (Thermo Fisher Cat. \# 4453320 Assay ID: Hs01110250_ m1 and Thermo Fisher Cat. \# 4331182, Assay ID: Hs01060665_g1) and TaqMan universal PCR master mix in a Bio-Rad CFX96 Real-Time system. Bio-Rad CFX Manager 2.0 software was used for analysis and HO-1 mRNA expression was normalized to $\beta$-actin and evaluated using one-way ANOVA from GraphPad Prism 7. The means \pm SD was plotted as fold change for each treatment group $(n=5)$.

\section{Fluorescence tracking and image analysis after photoconversion}

All images were captured using a Nikon A1R confocal microscope at excitation/emission wavelengths of 405/425-475 nm (for photoconversion and Hoechst nuclear stain), 488/505-550 nm for the green channel (Dendra2 before photoconversion and to visualize GFP-PML-I), 561/570-620 nm for the red channel (Dendra2 after photoconversion) and 640/662-737 $\mathrm{nm}$ for the far-red channel (Crimson-PML-I and Crimson-PML-I-2KR). We measured the maximum energy output during photoconversion using the X-cite XR2100 Power meter. The range of power output during photoconversion of the $405 \mathrm{~nm}$ laser was 12.2$62.7 \mu \mathrm{W}$. We calculated the power $/$ pixel $=1.49 \mathrm{nW} / \mathrm{px}$ using the maximum power detected. Fluorescence intensities were measured in specified regions of interest using the ROI editor in NIS Elements Advanced Research 4.0 microscope imaging software. Quantitative data was exported into Microsoft Excel.

Background fluorescence intensity values in the red channel of non-transfected HepG2 cells were obtained using the same camera settings and set-up. These were subtracted from the raw fluorescence intensities in the red channel of cells expressing Dendra2 or Dendra2-Nrf2 during the course of the experiment. The average fluorescence intensity in the red channel before photoconversion was calculated. The post-photoconversion values were normalized to the pre-photoconversion values. To eliminate the initial fluctuations in fluorescence, normalized values at 5 minutes post-photoconversion were set to 100 and the subsequent fluorescence intensities were normalized to this value. The relative fluorescence intensity was plotted over time and displayed graphically using GraphPad Prism 7 one-phase decay. The average decay rate constants and half-lives were calculated using PyFDAP v1.1.2 software as previously reported [23, 24] with minor modifications. Briefly, the normalized post-photoconversion fluorescence intensity values were imported into PyFDAP v1.1.2 as a .csv file. The "noise" value was determined to be 40 by taking images using the same parameters and set-up in the absence of a culture dish. The data was plotted using a "fit" of the average decay pattern from the individual cells. The mathematical equations used to derive the decay parameters are: $c(t)=c_{0} e^{-k t}+y_{0}$ where $c(t)$ is the concentration of a molecule at time $t, c(0)=c_{0}$ is the concentration at time $t=0$, and $y_{0}$ is the baseline fluorescence intensity to which the population of decaying molecules converge and $\mathrm{k}=$ decay rate. From $\mathrm{k}$ we computed the half-life $\tau$ using the equation $\tau=\ln (2) / \mathrm{k}[23,24]$.

\section{KARGER}




\section{Cellular Physiology Cell Physiol Biochem 2018:47:800-816 \begin{tabular}{l|l|l} 
and Biochemistry & $\begin{array}{l}\text { DOI.1159/000490033 } \\
\text { Published online: May 28, } 2018\end{array}$ & $\begin{array}{l}\text { () 2018 The Author(s). Published by S. Karger AG, Basel } \\
\text { www.karger.com/cpb }\end{array}$
\end{tabular}

\section{Results}

\section{Assessment of expression and photoconversion of Dendra2-Nrf2 in HepG2 cells}

To use the photoconversion property of Dendra2 to study the dynamics of Nrf2 in live cells, HepG2 cells were transiently transfected with plasmids expressing Dendra2 or Dendra2-Nrf2. The expression of Dendra2-Nrf2 was detected by western blot analysis using either anti-Nrf2 antibody (Fig. 1A, left panel) or anti-Dendra2 antibody (Fig. 1A, right panel). To demonstrate photoconversion, a $405 \mathrm{~nm}$ laser $(0.5 \mathrm{sec})$ was applied to HepG2 cells expressing Dendra2, GFP-Nrf2 or Dendra2-Nrf2. In Fig. 1B photoconversion of HepG2 cells expressing either Dendra2 (top row) or Dendra2-Nrf2 (bottom row) exhibited a change in color from green to red while photoconversion of cells expressing GFP-Nrf2 (middle row) did not result in a similar change in color because GFP is not a natural photoconvertible fluorescent protein [20]. In a similar experiment, arbitrarily selected regions (white circles, top row) within HepG2 cells expressing Dendra2, GFP-Nrf2 or Dendra2-Nrf2 were photoconverted and monitored over time. The real-time change in color and rapid redistribution of photoconverted Dendra2 (left column) or Dendra2-Nrf2 (right column) away from the indicated regions is illustrated in Fig. 1C. As expected, application of the $405 \mathrm{~nm}$ laser to HepG2 cells expressing GFP-Nrf2 did not result in any change in color. Green and red fluorescence intensities, normalized to pre-photoconversion values, at the point of application of the laser (1C, white circles, top row) were quantified and displayed

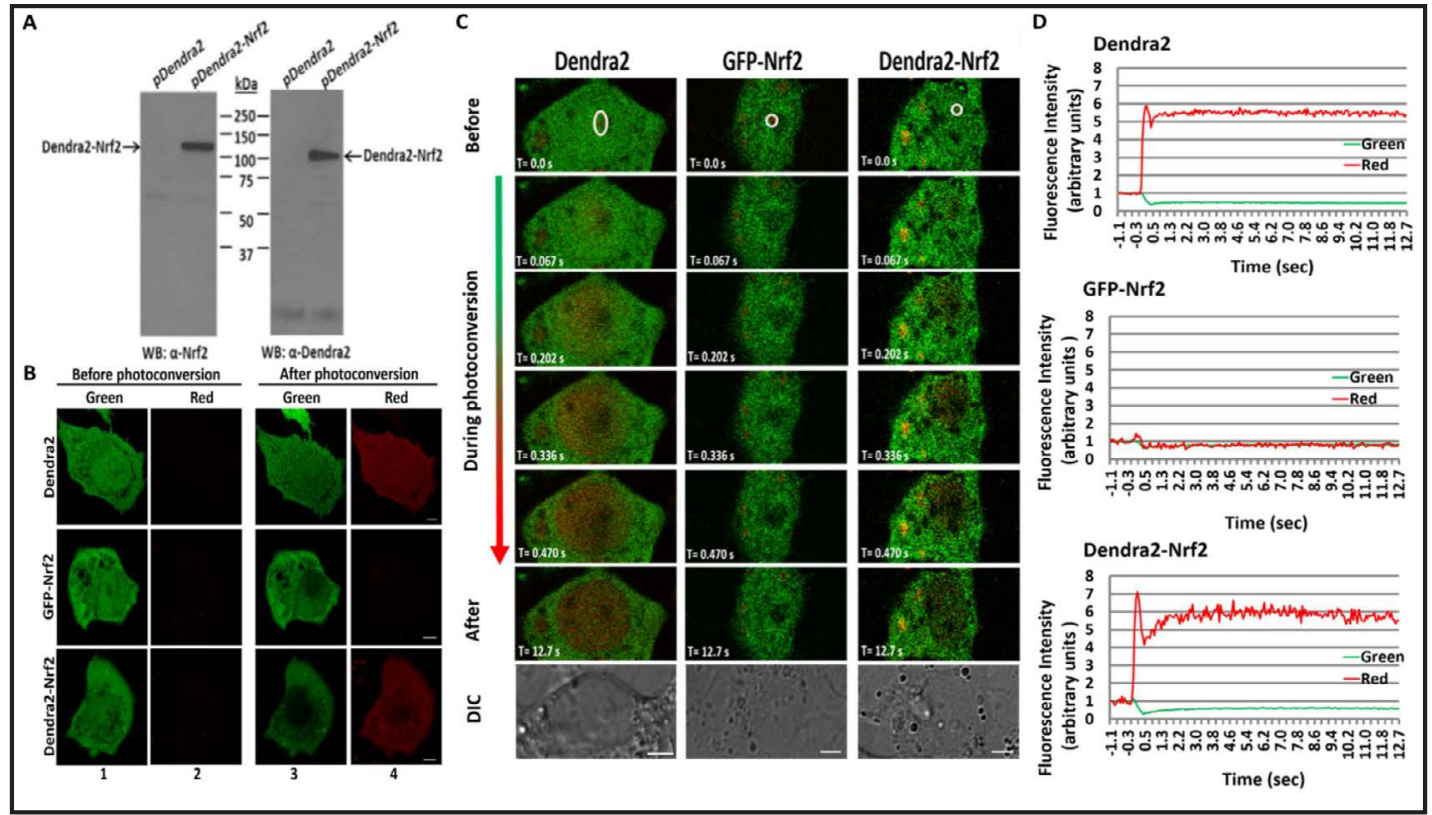

Fig. 1. Assessment of expression and photoconversion of Dendra2-Nrf2 in HepG2 cells. A, HepG2 cells (1 x $10^{6}$ ) seeded in $100 \mathrm{~mm}$ dishes were transfected with $5 \mu \mathrm{g}$ of pDendra 2 or pDendra2-Nrf2. After $48 \mathrm{~h}$, whole cell lysates $(40 \mu \mathrm{g})$ were separated by $8 \%$ SDS-PAGE and immunoblotted with antibodies against Nrf2 (left panel) or Dendra2 (right panel). B, Confocal images before and after photoconversion. HepG2 cells (5 x $\left.10^{5}\right)$ were transfected with $1 \mu \mathrm{g}$ of pDendra2 (top row), pGFP-Nrf2 (middle row) or pDendra2-Nrf2 (bottom row) in glass bottom MatTek dishes and visualized $48 \mathrm{~h}$ later. Photoconversion was performed by applying a 405$\mathrm{nm}$ laser for $0.5 \mathrm{sec}$ to an arbitrary region within the cell; images were acquired directly after photoconversion. C, Demonstration of the process of photoconversion in real time. HepG2 cells expressing Dendra2 (left column), GFP-Nrf2 (middle column) or Dendra2-Nrf2 (right column) were cultured and photoconverted as in B in the indicated regions (white circles, top row). Shown are merged images from those taken in the green and red channels at the indicated time points. D, Graphic representation of the average fluorescence intensities measured in the green and red channels at the point of photoconversion (measured within the white circles in C, top row) of cells expressing Dendra2, GFP-Nrf2 and Dendra2-Nrf2 ( $\mathrm{n}=12$-14 cells per group). Scale bars, $5 \mu \mathrm{m}$. 
graphically in Fig. 1D. These data suggest that fusion of Dendra2 to Nrf2 does not impair photoconversion of Dendra2 in HepG2 cells.

\section{Dendra2-Nrf2 functionally mimics native Nrf2}

We used two approaches to demonstrate that Dendra2-Nrf2 behaves like native Nrf2. In the first approach, we monitored the effect of Dendra2-Nrf2 on tBHQ-dependent induction of heme oxygenase-1 (HO-1) expression. It is well-established that the electrophile tBHQ activates endogenous $\mathrm{Nrf} 2[1,25]$, resulting in induction of Nrf2-regulated genes such as HO-1. HepG2 cells transiently expressing Dendra2-Nrf2 followed by an $8 \mathrm{~h}$ treatment with tBHQ resulted in induction of mRNA encoding HO-1 (Fig. 2A) and protein (Fig. 2B) consistent with the expected action of tBHQ on Nrf2-regulated genes [1].

In the second approach, we treated HepG2 cells stably expressing Dendra2-Nrf2 with SN50, a cell-penetrating synthetic peptide to block nuclear translocation of Nrf2 [22]. Consistent with previous reports on the inhibition of nuclear transport of other stressresponsive transcription factors such as NF- $\mathrm{kB}$ (nuclear factor kappa-light-chain-enhancer of activated B cells), NFAT (nuclear factor of activated T-cells), AP-1(activator protein 1) and

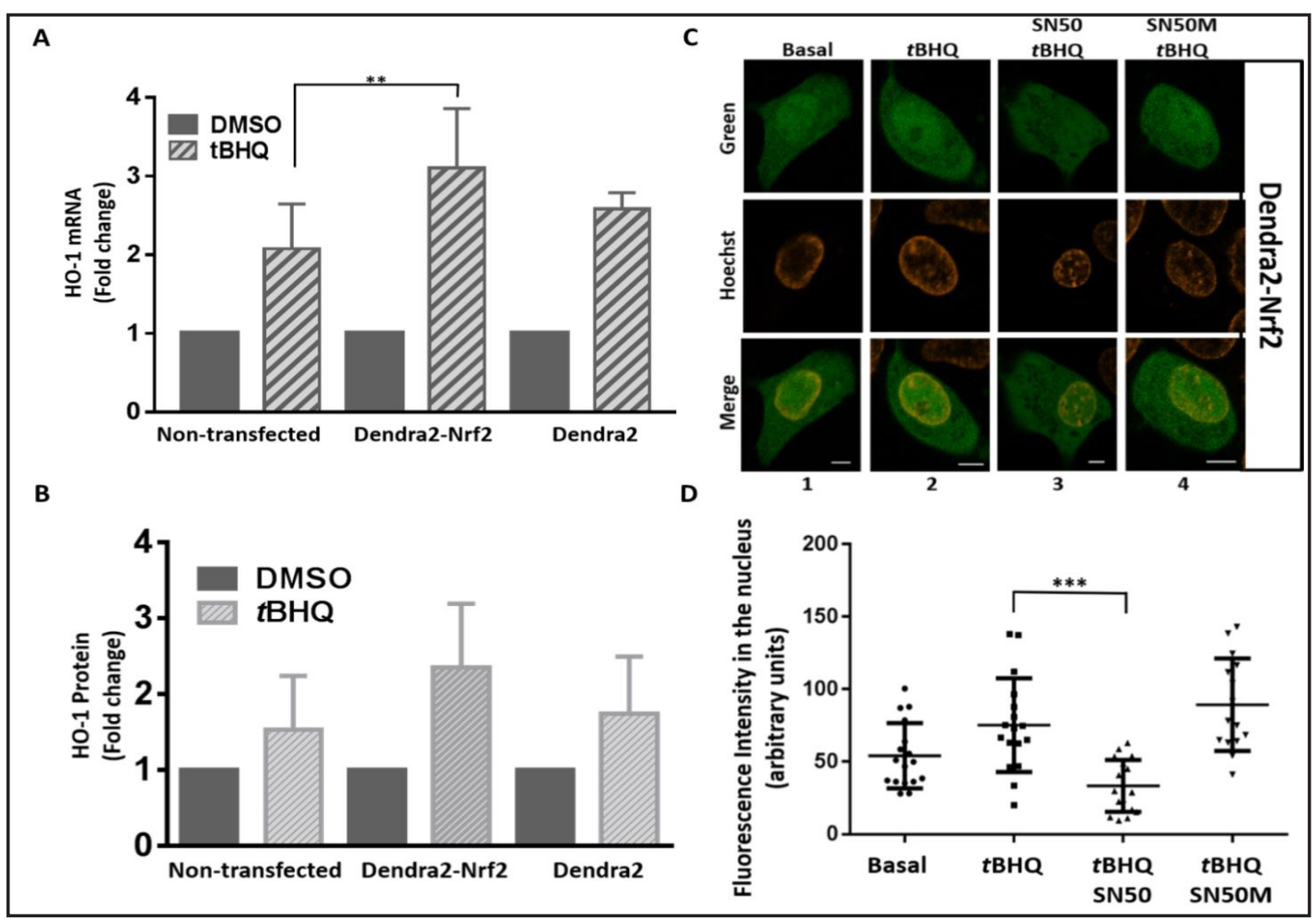

Fig. 2. Dendra2-Nrf2 functions like native Nrf2. A-B, effect of tBHQ on Dendra2-Nrf2-mediated expression of heme oxygenase-1 (HO-1). HepG2 cells $\left(5 \times 10^{5}\right)$ were transfected with $2.5 \mu \mathrm{g}$ of pDendra2 or pDendra2Nrf2 and grown for $48 \mathrm{~h}$. The cells were then treated with tBHQ $(20 \mu \mathrm{M})$ for $8 \mathrm{~h}$. Total mRNA and whole cell lysates were processed for RT-PCR and western blot analyses, respectively. A, HO-1 mRNA expression was normalized to $\beta$-actin and evaluated using one-way Anova from GraphPad Prism 7. The mean \pm SD was plotted as fold change for each treatment group $(n=5) .{ }^{* *} p<0.01 B$, Densitometric analysis of HO-1 protein expression. Bars represent relative HO-1 expression (mean $\pm \mathrm{SD}, \mathrm{n}=6$ ) normalized to $\mathrm{LDH}$ loading control and relative to DMSO control. C-D, SN50 inhibits tBHQ-induced translocation of Dendra2-Nrf2 into the nucleus. C, HepG2 cells $\left(5 \times 10^{5}\right)$ stably expressing Dendra2-Nrf2 were grown for $48 \mathrm{~h}$. Cells were then pre-treated with either SN50 $(50 \mu \mathrm{M})$ or SN50M $(50 \mu \mathrm{M})$ for $1 \mathrm{~h}$ followed by treatment with tBHQ $(20 \mu \mathrm{M})$ for $1 \mathrm{~h}$. Hoechst 33342 stain was added to the culture dish to visualize the nucleus (405 nm channel- colored orange). D, the expression of Dendra2-Nrf2 (green fluorescence intensity) in the nucleus was plotted for each group ( $\mathrm{n}=15-17$ cells per group) and evaluated using one-way Anova from GraphPad Prism 7. $* * * \mathrm{p}<0.001$. Scale bars, $5 \mu \mathrm{m}$. 


\section{Cellular Physiology Cell Physiol Biochem 2018;47:800-816

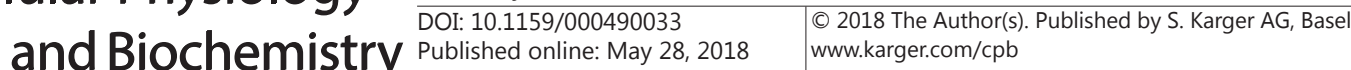 Burroughs et al.: PML-Nuclear Bodies Regulate the Stability of Nrf2}

STAT1 (signal transducer and activator of transcription 1) [26-29], SN50 blocked tBHQinduced nuclear accumulation of Dendra2-Nrf2 (Fig. 2C, compare columns 2 and 3). The scrambled peptide SN50M (which penetrates cells but does not interfere with nuclear translocation of Nrf2) had no effect. Quantification of the imaging data is shown in Fig. 2D. Together, the data in Fig. 2 suggest that fusion of Dendra2 to Nrf2 did not interfere with nuclear translocation or transcriptional activity of Nrf2 in response to oxidative stress.

Use of photoconversion of Dendra2-Nrf2 to monitor degradation of Nrf2 in whole cells

Green fluorescence exhibited by Dendra2 or Dendra2-Nrf2 reflects steady-state levels of these proteins whereas red fluorescence represents previously synthesized protein that has undergone irreversible photoconversion. Therefore, monitoring decay of red fluorescence over time provides real-time measurements of degradation of Nrf2 in living cells as opposed to reagents that block global protein synthesis, such as cycloheximide, which interferes with other cellular processes.

To determine the impact of photoconversion on cell viability, we simulated photoconversion by applying a $405 \mathrm{~nm}$ laser for $2.0 \mathrm{sec}$ to an area encircling non-transfected HepG2 cells and measured the percentage of viable cells (see online suppl. material, Fig. S1). More than $58 \%$ of cells remained alive $3 \mathrm{~h}$ after photoconversion at the maximum setting. With the exception of Fig. 3, all photoconversion experiments were performed using sub-maximal exposure times to the $405 \mathrm{~nm}$ laser $(0.5 \mathrm{sec})$. Using the same parameters, we photoconverted single cells expressing Dendra2-Nrf2 (Fig. 3A) or Dendra2 (Fig. 3C) and show representative images depicting changes in green (non-photoconverted) and red (photoconverted) fluorescence intensities over the duration of the experiment. Only cells that retained their initial morphology, indicative of live cells, were used in quantitative analyses.

To quantify the change in red fluorescence after photoconversion, the average red fluorescence intensities were plotted over time with error bars representing the standard

Fig. 3. Monitoring degradation of Dendra2-Nrf2 under basal and induced conditions in single live cells. HepG2 cells $\left(5 \times 10^{5}\right)$ stably expressing Dendra2-Nrf2 (A) or Dendra2 (C) were seeded on glass bottom MatTek dishes for $48 \mathrm{~h}$ later. Prior to visualization, some cells were pre-treated with tBHQ $(20 \mu \mathrm{M})$ for $2 \mathrm{~h}$ or MG-132 $(10 \mu \mathrm{M})$ before photoconversion. Single cells expressing Dendra2Nrf2 or Dendra2 were de-
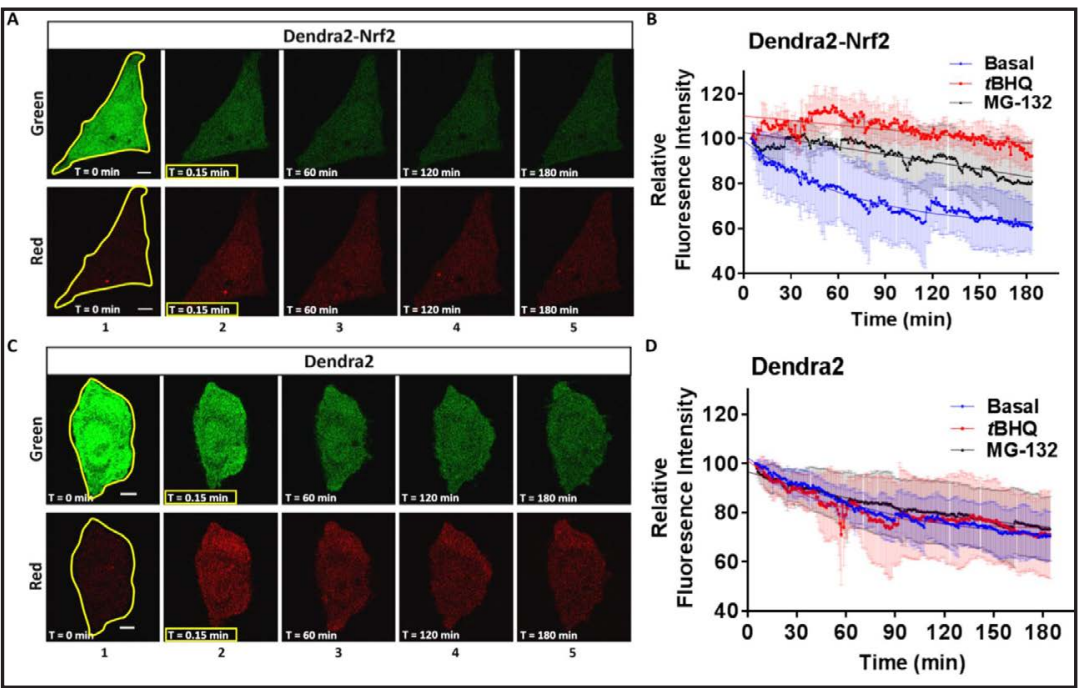

marcated around the cell

margin by the auto-detection feature of NIS Elements Advanced Research 4.0 software and photoconverted using a $405 \mathrm{~nm}$ laser for 2.0 sec to the indicated area. Representative image panels of single cells expressing Dendra2-Nrf2 or Dendra2 under basal conditions (no treatment) is illustrated in panels A and C, respectively. Time lapse images were captured every 45 seconds in the green and red channels after photoconversion and processed as described in materials and methods. Any change(s) in morphology and position of the cells over time was adjusted by the imaging instrument using NIS Elements Advanced Research 4.0 software. The average fluorescence intensity for Dendra2-Nrf2 (n= 5-7) or Dendra2 (n= 5-22) was plotted over time using one-phase decay analysis of GraphPad Prism 7 with error bars representing the standard deviation. Scale bars, $5 \mu \mathrm{m}$. 
deviation from cells expressing Dendra2-Nrf2 (Fig. 3B) or Dendra2 (Fig. 3D). Under basal conditions, the $t_{1 / 2}$ of Dendra2-Nrf2 was 35 minutes, consistent with previous reports $[11,12$, $30,31]$. However, treatment of cells with tBHQ to induce stress conditions led to stabilization of $\mathrm{Nrf} 2$ and the $t_{1 / 2}$ was dramatically extended by $>28$ fold or 1, 006 minutes (Fig. 3B and Table 1). The decay rate of Dendra2-Nrf2 in the presence of tBHQ was statistically different ( $p<0.01$ ) from the decay rate under basal conditions (Fig. 4). To establish that decay of photoconverted Dendra2-Nrf2 represents degradation of Nrf2, HepG2 cells expressing Dendra2-Nrf2 were treated with MG-132 to inhibit proteasome function. This treatment altered the slope of the decay curve and extended the $t_{1 / 2}$ to 231 minutes. This indicates that decay of red fluorescence of Dendra2-Nrf2 occurs via the proteasome and is not a result of inadvertent photobleaching. Under basal conditions, the $t_{1 / 2}$ of Dendra2 alone was 59 minutes. Treatment with $\mathrm{tBHQ}$ or MG-132 had little effect on the decay pattern of Dendra2 compared to basal conditions (Fig. 3D and Fig.4). Taken together, these results provide proof that the photoconversion paradigm can be used to measure degradation of Nrf2 in single live cells.

Dendra2-Nrf2 is degraded at a slower rate in the nucleus

We previously showed that Nrf2 translocates into the nucleus in response to pro-oxidant stimuli and contains multiple nuclear localizations signals [22]. Additionally, Nrf2 harbors nuclear export signals [32, 33] allowing Nrf2 to traverse the nuclear membrane. To determine the fate of the nuclear sub-population of Nrf2, without impairing nuclear export, we photoconverted Dendra2-Nrf2 within the nucleus (Fig. 5A, column 1 , yellow circle) and tracked the decay of red fluorescence over time. Acknowledging the limitation that Dendra2-Nrf2 can traffic throughout the cell, we measured the decay rate in the nucleus (Fig. 5B) and the nuclear derived Dendra2-Nrf2

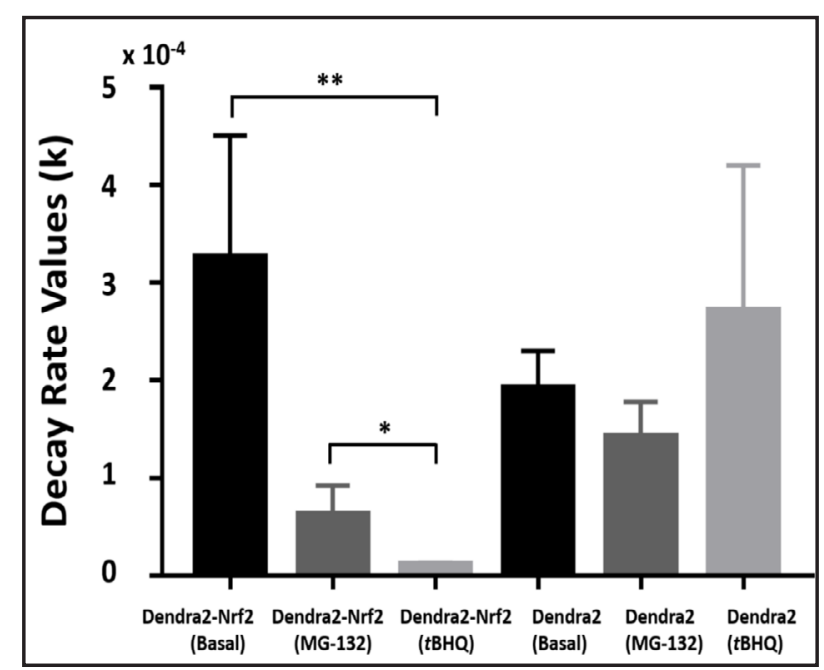

Fig. 4. Mean Decay rate values of Dendra2-Nrf2 and Dendra2. Red fluorescence after photoconversion was monitored over time in individual cells expressing Dendra2-Nrf2 or Dendra2. The red fluorescence decay of each cell was fit to the average exponential decay curve within the treatment group and the decay rate value $(\mathrm{k})$ was determined. Average $\mathrm{k}$ values for each treatment group are shown. Average $\mathrm{k}$ values that are significantly different between different experimental groups ( $\mathrm{p}$-value ${ }^{* *}<0.01$ and ${ }^{*} \mathrm{p}<0.05$, Wilcoxon Mann-Whitney test) are indicated. Larger $\mathrm{k}$ values indicate higher rates of red fluorescence decay. Error bars indicate standard error of the mean (SEM).

Table 1. Average decay parameters of Dendra2-Nrf2 and Dendra2 using the photoconversion technique. The average half-life and decay rate values of Dendra2 and Dendra2-Nrf2 were calculated using PyFDAP v1.1.2 software

\begin{tabular}{lcc}
\hline Dendra2-Nrf2 & $\begin{array}{c}\text { Half-life } \\
\left(\mathrm{t}_{1 / 2}\right) \mathrm{min}\end{array}$ & Avg. Decay Rate $(\mathrm{k})$ \\
\hline Basal & 35 & $3.2 \times 10^{-4}$ \\
MG-132 $(10 \mu \mathrm{M})$ & 231 & $1.1 \times 10^{-5}$ \\
tBHQ $(20 \mu \mathrm{M})$ & 1,006 & $5.0 \times 10^{-5}$ \\
\hline Dendra2 & & \\
\hline Basal & 59 & $1.9 \times 10^{-4}$ \\
MG-132 $(10 \mu \mathrm{M})$ & 53 & $2.1 \times 10^{-5}$ \\
tBHQ $(20 \mu \mathrm{M})$ & 81 & $1.4 \times 10^{-5}$ \\
\hline
\end{tabular}


Table 2. Average decay parameters of photoconverted nuclear Dendra2-Nrf2 measured in the nucleus and whole cell. The average half-life and decay rate values of Dendra2-Nrf2 were calculated using PyFDAP v1.1.2 software

\begin{tabular}{lcccc}
\hline & \multicolumn{2}{c}{ Nucleus } & \multicolumn{2}{c}{ Whole } \\
& $\begin{array}{c}\text { Half-life } \\
\left(\mathrm{t}_{1 / 2}\right) \text { min }\end{array}$ & $\begin{array}{c}\text { Avg. Decay } \\
\text { Rate }(\mathrm{k})\end{array}$ & $\begin{array}{c}\text { Half-life } \\
\left(\mathrm{t}_{1 / 2}\right) \text { min }\end{array}$ & $\begin{array}{c}\text { Avg. Decay } \\
\text { Rate }(\mathrm{k})\end{array}$ \\
\hline- & 66 & $1.7 \times 10^{-4}$ & 80 & $1.4 \times 10^{-4}$ \\
Crimson-PML-I & 51 & $2.2 \times 10^{-4}$ & 137 & $8.4 \times 10^{-5}$ \\
Crimson-PML-I-2KR & 45 & $2.5 \times 10^{-4}$ & 345 & $3.3 \times 10^{-5}$ \\
\hline
\end{tabular}

Fig. 5. Dendra2-Nrf2 is degraded in the nucleus of single live cells. HepG2 cells $\left(5 \times 10^{5}\right)$ expressing Dendra2-Nrf2 were seeded on glass bottom MatTek dishes and visualized 48 $\mathrm{h}$ later. Prior to photoconversion, cells were pretreated without or with Leptomycin B (10 ng/ml) for $5 \mathrm{~h}$ to inhibit nuclear export (A and $C$ ). The nucleus was outlined as indicated and photoconverted using a $405 \mathrm{~nm}$ laser for $0.5 \mathrm{sec}$. Time-lapse images for untreated cells (A) and Leptomycin B treated (C)

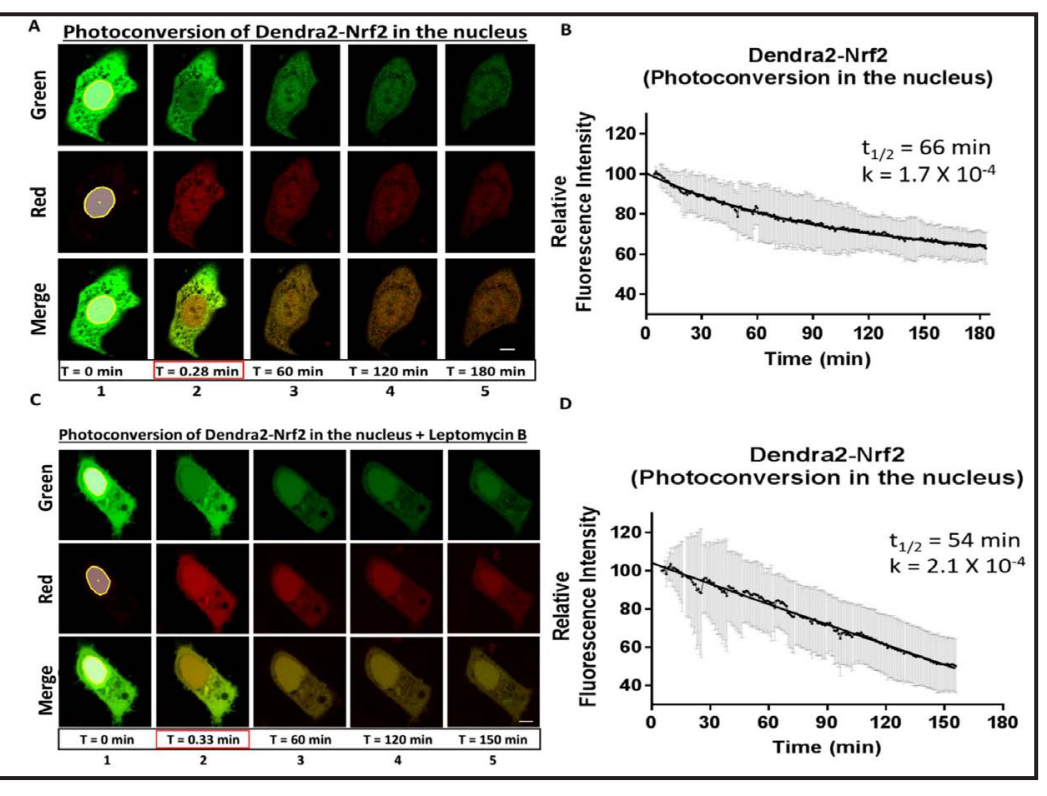
cells were captured every

45 seconds in the green and red channels after photoconversion. Oscillations in the relative fluorescence intensity are an artifact of manual refocusing of the microscope in D, but do not change the average decay pattern of red fluorescence. The average fluorescence decay after photoconversion in the red channel was quantified in the nucleus in the untreated (B) or in the Leptomycin B treated (D) cells and plotted over time as described in Fig. 3. Scale bars, $5 \mu \mathrm{m}$.

in the whole cell (Table 2). Under basal conditions, the decay of photoconverted nuclear Dendra2-Nrf2, measured in the nucleus, had a $t_{1 / 2}$ of 66 min while the $t_{1 / 2}$ measured in the whole cell was $80 \mathrm{~min}$. The different half-lives of the nuclear subset of Dendra2-Nrf2 could indicate compartment-specific regulation of degradation of Nrf2. Similar experiments were performed using Dendra2 and the half-life of photoconverted nuclear Dendra2 (see online suppl. material, Fig. S2 and Table S2) was comparable to that of Dendra2 photoconverted in the whole cell (Fig. 3).

In order to measure degradation of Nrf2 within the nucleus, HepG2 cells expressing Dendra2-Nrf2 were treated with Leptomycin B, an inhibitor of exportin 1/CRM1 protein, and the nuclear sub-population of Dendra2-Nrf2 was photoconverted (Fig. 5C, column 1, yellow circle). The majority of red fluorescence was concentrated in the nucleus and decayed over time with a $t_{1 / 2}$ of 54 min (Fig. 5D). While Leptomycin B blocked a substantial amount of nuclear export, it is possible that other uninhibited karyopherins exported Dendra2-Nrf2 out of the nucleus, resulting in red fluorescence in the cytoplasm. Altogether, these results provide direct evidence that Nrf2 is degraded in the nucleus and signify differential rates of degradation of the nuclear subset of Nrf2 in HepG2 cells.

Assessment of the role of PML nuclear bodies in degrading Dendra2-Nrf2 in the nucleus

We and others have shown that Nrf2 can traffic to PML-NBs [34, 35], which are nuclear domains that regulate posttranslational modifications of partner proteins 


\section{Cellular Physiology Cell Physiol Biochem 2018;47:800-816 and Biochemistry Published online: May 28, $2018 \quad \begin{aligned} & \text { DOI: 10.1159/000490033 } 2018 \text { The Author(s). Published by S. Karger AG, Basel } \\ & \text { www.karger.com/cpb }\end{aligned}$ \\ Burroughs et al.: PML-Nuclear Bodies Regulate the Stability of Nrf2}

through phosphorylation, acetylation, sumoylation [post-translational modification by small ubiquitin-like modifier (SUMO) proteins] and ubiquitylation [36]. These modifications can lead to activation, sequestration or degradation of recruited proteins having profound effects on biological processes such as: transcription, apoptosis, DNA repair, and stem cell self-renewal [36].

To better understand the role of PML-NBs on the stability of Nrf2 in the nucleus, using the photoconversion paradigm, we established a plasmid expressing Crimson-PML-I fusion protein. Given that a PML specific antibody cannot discriminate the endogenous from the recombinant PML, we opted to colocalize Crimson-PML-I and GFP-PML. The expression of Crimson-PML-I fusion protein was verified by western blot analysis using either anti-PML (Fig. 6A, left panel) or anti-Crimson (Fig. 6A, right panel) antibodies. To validate the utility of Crimson-PML-I, we used immunofluorescence to detect the cellular locale of endogenous PML (Fig. 6B, column 1) compared to Crimson-PML-I (Fig. 6B, column 3). The endogenous PML and Crimson-PML-I had a similar cellular distribution. Because GFP-PML-I has been demonstrated to co-localize with endogenous PML [37], we confirmed co-localization of Crimson-PML-I and GFP-PML-I in cells co-transfected with plasmids encoding these fusion proteins (Fig. 6B, column 4). Regions where fluorescence in the green and far-red channels overlap were confirmed by the graphic display in Fig. 6C and see online suppl. material, Fig. S3, suggesting co-localization of these two proteins in PML-NBs.

Next, we co-expressed Crimson-PML-I in cells stably expressing Dendra2-Nrf2 and photoconverted the nucleus to track the decay of red fluorescence in various sub-cellular regions. Fig. 7A illustrates fluorescence of Dendra2-Nrf2 (green, column 1), photoconverted Dendra2-Nrf2 (red, column 2) and Crimson-PML-I (far-red, colored blue, column 3) directly after photoconversion in three distinct cells. Fig. 7A columns 4 and 5 display merged images (overlap of green, red and far-red channels) of cells co-expressing Dendra2-Nrf2 and Crimson-PML-I after photoconversion. Regions where Dendra2-Nrf2 and Crimson-PML-I co-localize can be visualized as purple-colored dots (Fig. 7 A column 4) or projections/spikes

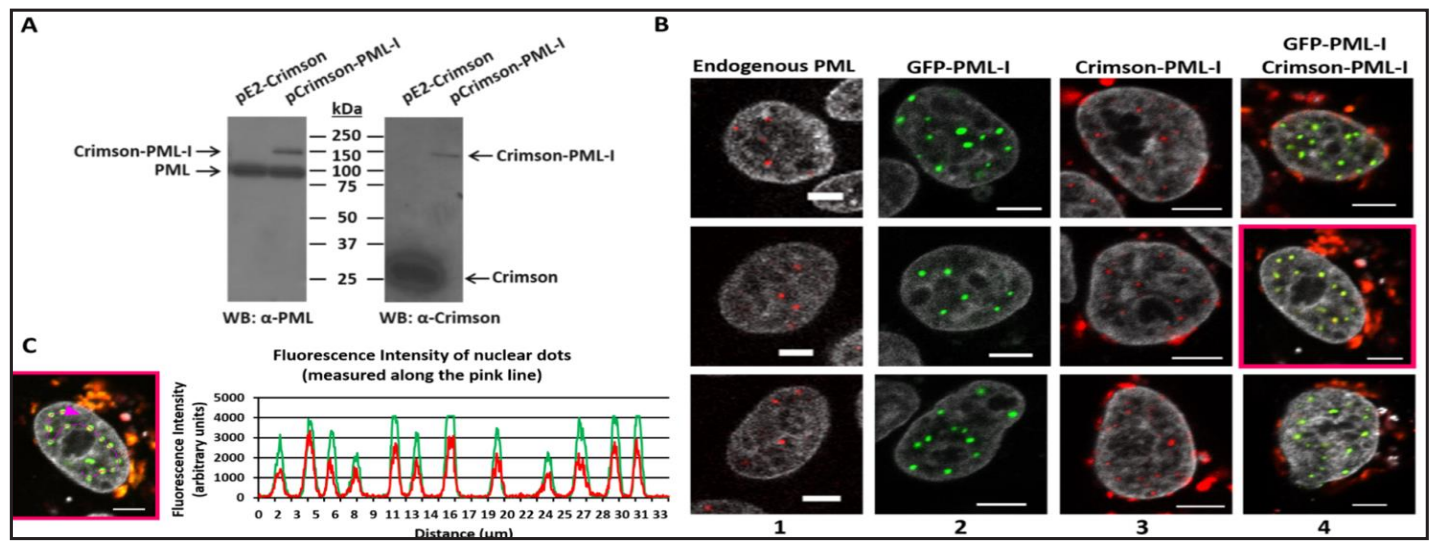

Fig. 6. Expression of Crimson-PML-I fusion protein and co-localization with GFP-PML-I. A, HepG2 cells (5 x $10^{5}$ ) were transfected with $2.5 \mu$ g of plasmids expressing pE2-Crimson (empty vector) or pCrimson-PML-I encoding the fusion protein Crimson-PML-I. After $48 \mathrm{~h}$, whole cell lysates (30 $\mu \mathrm{g}$ ) were separated by $8 \%$ SDS-PAGE and immunoblotted using anti-PML and anti-Crimson antibodies. B, HepG2 cells $\left(5 \times 10^{5}\right)$ were non-transfected (column 1) or transfected with $0.25 \mu$ g of pGFP-PML-I (column 2) or $0.5 \mu \mathrm{g}$ of pCrimsonPML-I (column 3) or co-transfected with $0.25 \mu \mathrm{g}$ of pGFP-PML-I and $0.5 \mu \mathrm{g}$ of pCrimson-PML-I, fixed, and visualized by confocal microscopy. The non-transfected cultures were processed for immunofluorescence to detect endogenous PML as described in "Experimental Procedures." The image panels represent cellular localization of: endogenous PML protein detected by immunofluorescence, GFP-PML-I detected in the green channel, Crimson-PML-I detected in the far-red channel and co-localized GFP-PML-I and Crimson-PML-I merged, respectively. C, Fluorescence intensities measured along the pink line in the nucleus highlighted in B (column 4- middle row) demonstrate co-localization of GFP-PML-I and Crimson-PML-I in the green and far-red channels. Scale bars, $5 \mu \mathrm{m}$. 


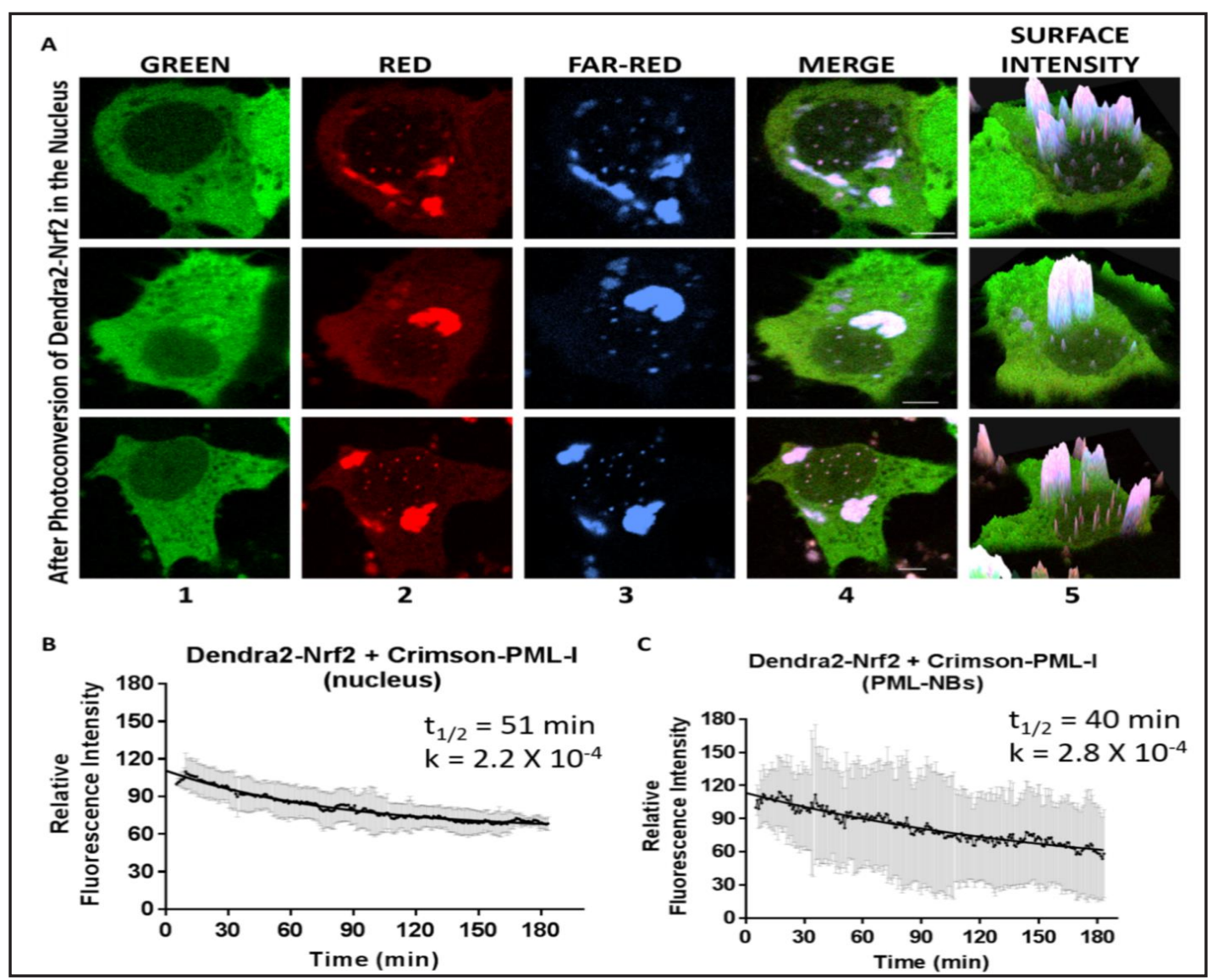

Fig. 7. Monitoring degradation of Dendra2-Nrf2 in PML-NBs. A, HepG2 cells (5 x $\left.10^{5}\right)$ stably expressing Dendra2-Nrf2 were transfected with pCrimson-PML-I $(1 \mu \mathrm{g})$ in glass bottom MatTek dishes and visualized $48 \mathrm{~h}$ later. Prior to photoconversion of Dendra2-Nrf2, the nucleus was outlined and photoconverted using a $405 \mathrm{~nm}$ laser for $0.5 \mathrm{sec}$. Time-lapse images were captured in the green (column 1), red (column 2) and farred (column 3) channels after photoconversion. Merged images are shown in columns 4 and 5 to illustrate the cellular distribution of fluorescence within the live intact cells. B and C, the average fluorescence decay, after photoconversion, in the red channel was quantified in the nucleus (B) or in PML-NBs (C) (indicated by the pink dots in the nuclei of A-column 4) and plotted over time ( $\mathrm{n}=7$ cells, 25 PML-NBs). Scale bars, $5 \mu \mathrm{m}$.

(Fig. 7A column 5) within the nucleus. Up to ten such projections were evident per nucleus, which is consistent with previous studies in fixed cells that showed 5-15 PML-NBs per nucleus [36-40]. The decay of red fluorescence over time, generated from photoconverted Dendra2-Nrf2 in the nucleus, was measured in the nucleus, PML-NBs, nucleoplasm (regions in the nucleus lacking PML) and in the whole cell. A graphic display of the average red fluorescence intensities over time, measured in the nucleus and PML-NBs, are illustrated in Fig. 7B and C, respectively. The $t_{1 / 2}$ of photoconverted Dendra2-Nrf2, measured in the nucleoplasm, was 34 min while the $t_{1 / 2}$ measured in PML-NBs was $40 \mathrm{~min}$. The $t_{1 / 2}$ of photoconverted Dendra2-Nrf2 measured in the entire nuclear region was $51 \mathrm{~min}$. Given that Nrf2 can traffic out of the nucleus, the decay in red fluorescence could be due to degradation within these defined regions and/or trafficking to other areas within the cell. Analysis of the decay of red fluorescence in the whole cell, revealed a much longer half-life of Dendra2Nrf2 ( $t_{1 / 2} 137 \mathrm{~min}$ ) when co-expressed with Crimson-PML-I (Table 2). Parallel experiments were performed with Dendra2 as a control. The decay of red fluorescence, generated from photoconverted Dendra2 in the nucleus, measured in the nucleus, PML-NBs and whole cell were relatively similar (see online suppl. material, Fig. S4 and Table S2). 


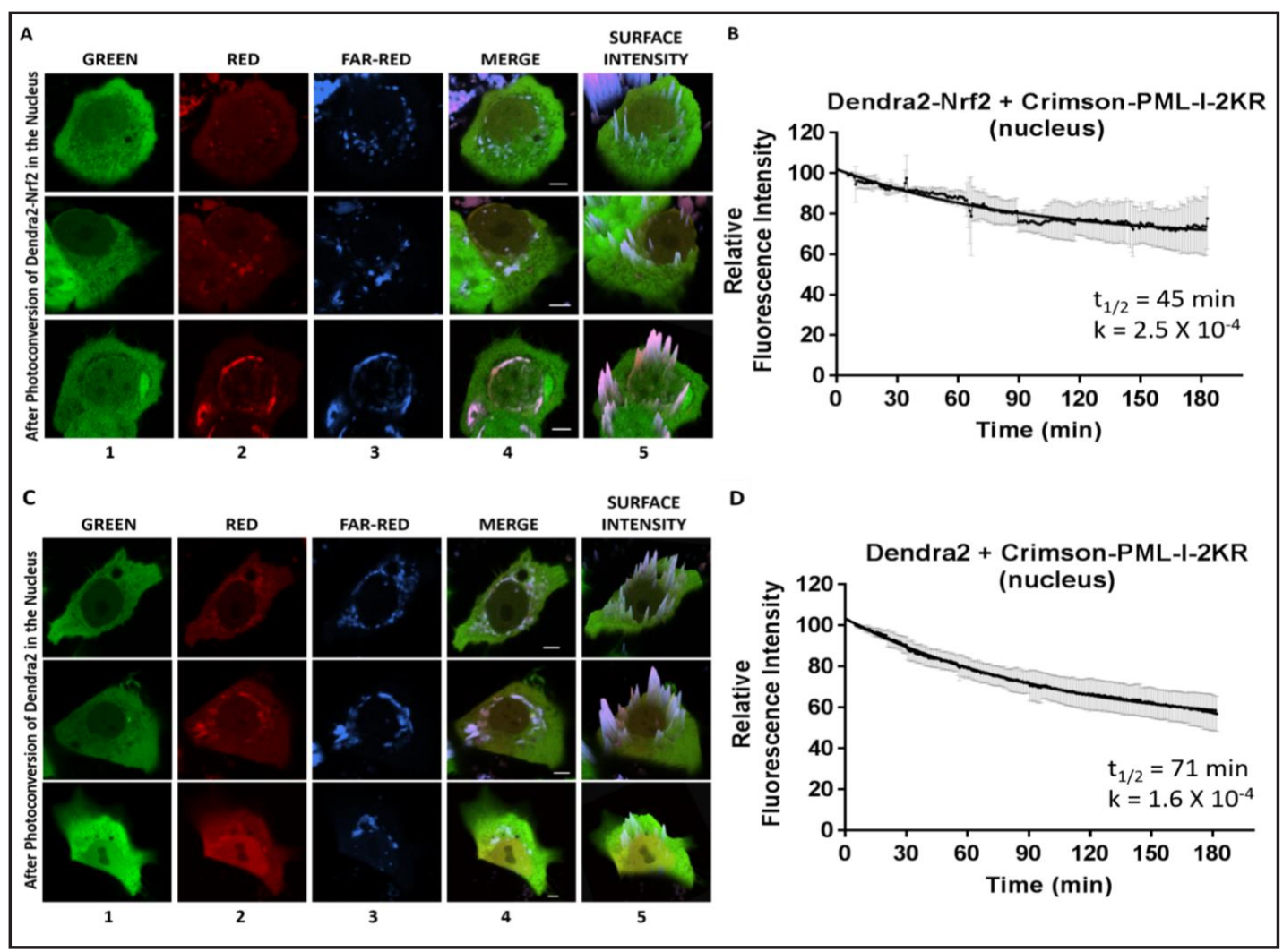

Fig.8. Monitoring degradation of Dendra2-Nrf2 in the nucleus of cells expressing SUMO-site mutant, Crimson-PML-I-2KR. A, HepG2 cells $\left(5 \times 10^{5}\right)$ stably expressing Dendra2-Nrf2 (A) or Dendra2 (C) were transfected with pCrimson-PML-I-2KR ( $1 \mathrm{~g}$ ) in glass bottom MatTek dishes and visualized $48 \mathrm{~h}$ later. The nucleus was outlined and photoconverted using a $405 \mathrm{~nm}$ laser for $0.5 \mathrm{sec}$. Time-lapse images were captured in the green (column 1), red (column 2) and far-red (column 3) channels after photoconversion. Merged images are shown in columns 4 and 5 to illustrate the cellular distribution of fluorescence within the live intact cells expressing Dendra2-Nrf2 (A) and Dendra2 (C), respectively. B and D, the average fluorescence decay, after photoconversion, in the red channel was quantified in the nucleus for Dendra2-Nrf2 (B) or Dendra2 (D) and plotted over time ( $n=5-6$ cells). Scale bars, $5 \mu \mathrm{m}$.

Monitoring the effect of SUMO-site mutant PML in degrading Dendra2-Nrf2 in the nucleus

PML is sumoylated on three target lysines K65, K160, and K490 [41]. While these SUMO sites are dispensable for nuclear body formation, K160 is also necessary for partner protein recruitment [42]. To allow for partner protein interaction, we created K65R and K490R mutations in the Crimson-PML-I plasmid (Crimson-PML-I-2KR) and monitored degradation of Dendra2-Nrf2 in cells co-expressing the SUMO-site-PML mutant. Under basal conditions, we observed a perinuclear distribution of Crimson-PML-I-2KR with a dramatic reduction in PML-NBs (1-5 per nucleus) (Fig. 8A and 8C column 3). In order to monitor degradation of Nrf2 without inadvertently altering the stability of Nrf2, we did not induce stress to facilitate PML-NB formation. Upon photoconversion of Dendra2-Nrf2 in the nucleus of cells coexpressing Crimson-PML-I-2KR, we detected a rapid redistribution of red Dendra2-Nrf2 (Fig $8 \mathrm{~A}$, column 2). Consequently, the average decay of red fluorescence, measured in the nucleus, revealed a $t_{1 / 2}$ of $45 \mathrm{~min}$ (Fig $8 \mathrm{~B}$ ). In contrast, the half-life of photoconverted Dendra2-Nrf2 derived from the nucleus, measured in the whole cell, was dramatically extended to $345 \mathrm{~min}$ (Table 2). Similar experiments using Crimson-PML-I-2KR were performed with Dendra2 as a control (Fig. 8C-D, and see online suppl. material, Table S2). Altogether, these data suggest that functional PML-NBs play an important role in regulating the stability of Nrf2. 


\section{Cellular Physiology Cell Physiol Biochem 2018;47:800-816

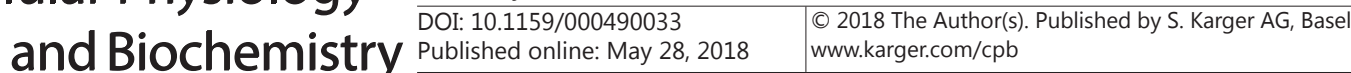 Burroughs et al.: PML-Nuclear Bodies Regulate the Stability of Nrf2}

\section{Discussion}

Nrf2 is a critical transcription factor that regulates hundreds of genes encoding a variety of proteins that function in diverse processes such as antioxidant defense, drug detoxification, cell growth, protein degradation and intermediary metabolism [6, 8, 4348]. As a transcription factor, it binds to the ARE to regulate the expression of several genes [1]. The prevailing model for regulating turnover of Nrf2 is that interaction of Nrf2 with the Keap1-Cul3-Rbx1 E3 ubiquitin ligase complex [9] leads to its ubiquitylation and degradation in proteasomes. Although functionally active Nrf2 is located in the nucleus, this Keap1-mediated degradation occurs primarily in the cytoplasm. The mechanism(s) for degrading Nrf2 in the nucleus and the time frame for its down regulation remains unclear. Because somatic mutations in Keap1 or Nrf2 can result in persistently high levels of Nrf2 [49-52], understanding regulatory processes that mediate degradation of Nrf2, especially under conditions of oxidative stress, could provide intriguing insight into the control of the cellular abundance of Nrf2. In this study, we developed and validated the use of the fusion protein Dendra2-Nrf2 to measure real-time degradation of Nrf2 in single live cells in the presence and absence of intact PML-NBs. Our data suggest that degradation of Nrf2 in the cytoplasm and nucleus is compartment specific and that intact PML-NBs are essential in the modification and degradation of nuclear Nrf2.

PML-NBs are assembled under conditions of oxidative stress and play a pivotal role in regulating stress-induced post-translational modifications of partner proteins [42]. In the absence of PML, ROS production is enhanced, Nrf2 protein is stabilized and this is accompanied by an increase in transcription of Nrf2-target genes [53]. Although defective mitochondrial activity has been indicated as a contributory factor for Nrf2 stabilization when PML is lacking, other explanations have been previously described. For example, Nrf2 can be degraded in PML-NB containing cell fractions [34], explaining subsequent stabilization of Nrf2 in the absence of PML. Further, in vivo studies using $\mathrm{PML}^{-1-}$ animals demonstrate a similar increase in Nrf2 regulated genes in liver tissues [54]. The activation of $\mathrm{Nrf2}$ is thought to be an adaptive response when PML is unavailable to modulate p53 activity [54]. These reports are consistent with our findings that PML plays a role in regulating the stability of Nrf2. Using the photoconversion paradigm, we demonstrated that Nrf2 is indeed degraded in the nucleus and the half-life of Nrf2 is not only compartment specific but also dependent on intact PML-NBs. This study also suggests that the rate of degradation of Nrf2

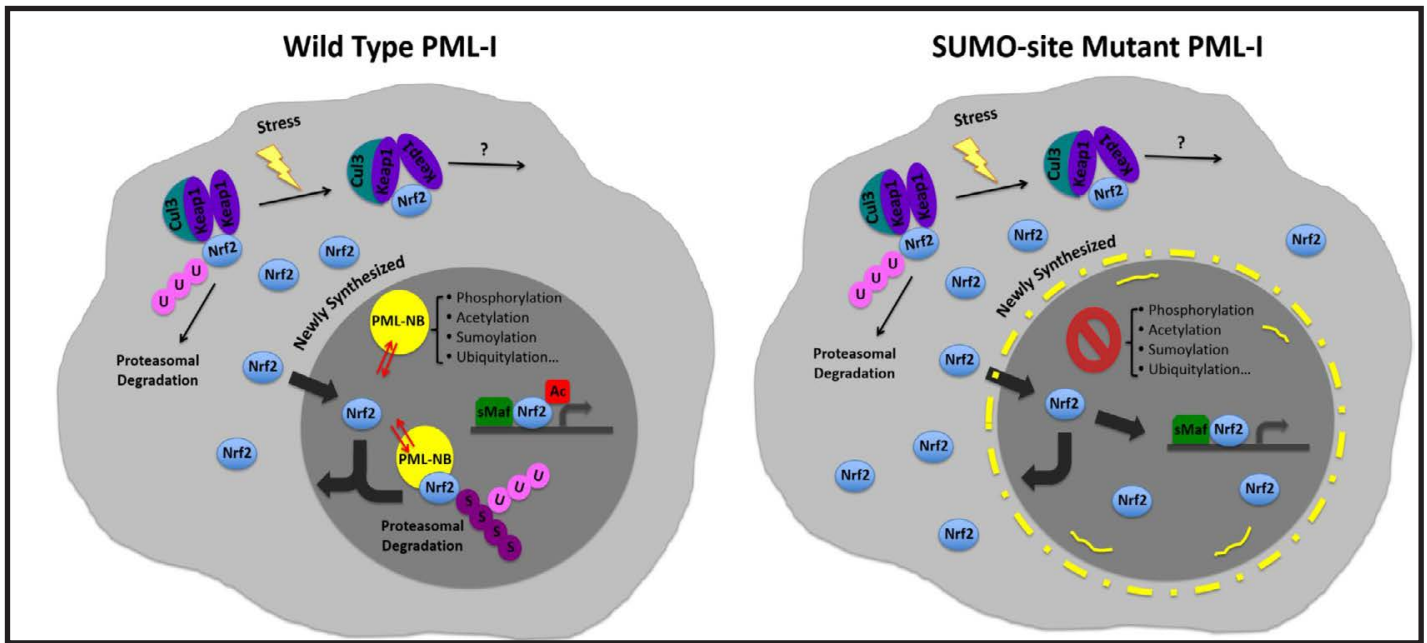

Fig. 9. Schematic depicting the role of PML-NBs in the regulation of Nrf2 stability in intact cells. In the presence of wild-type PML-I, Nrf2 is recruited to PML-NBs where it undergoes post-translational modifications that determine its fate (left). When PML-NBs are inefficiently formed or non-functional, Nrf2 may not undergo modifications required for its down regulation resulting in the stabilization of Nrf2 throughout the cell (right). 
in the nucleus may be influenced by the rate of translocation to other cellular compartments. Following inhibition of nuclear export, we observed that the rate of degradation of Dendra2Nrf2 in the nucleus is similar to that of degradation of Dendra2-Nrf2 in the nucleus of cells co-expressing Crimson-PML-I. However, expression of non-sumoylatable Crimson-PML-I resulted in a shorter half-life of Dendra2-Nrf2 in the nucleus but stabilized nuclear-derived Nrf2 in the whole cell.

Based on these data, we propose that Nrf2 can be recruited to PML-NBs and undergo post-translational modifications that lead to defined fate(s) of Nrf2. For example, sumoylated Nrf2 can be recognized by the SUMO targeted ubiquitin ligase, RNF4, which can facilitate its degradation [34]. However, when PML-NBs are poorly formed, the post-translational imprint on nuclear Nrf2 may be altered or absent and as such prevent Nrf2 from being down-regulated (Fig. 9).

Interestingly, we found the rate of decay of nuclear Dendra2-Nrf2 to be slower than that of Nrf2 in the whole cell and that some nuclear-derived Nrf2 trafficked to other cellular compartments. The extended $t_{1 / 2}$ of nuclear Dendra2-Nrf2 was more evident in cells expressing Crimson-PML-I and SUMO-site mutant Crimson-PML-I-2KR (Table 2). This extended half-life of nuclear Nrf2 may be due to compartment-specific differences in ROS levels, post-translational modifications, and/or nucleo-cytoplasmic trafficking. For example, the redox-insensitive Neh6 domain of Nrf2 contains a phosphodegron that mediates degradation of Nrf2 by the E3 ubiquitin ligase $\beta$-TrCP $[13,14,55]$, although the cellular locale remains under investigation. Other modes of repressing Nrf2 have also been described. In 2010, Kang et al [56]. showed that CRIF1 (CR6-interacting factor 1) interacts with Nrf2 and enhanced the ubiquitylation of Nrf2 under conditions of oxidative stress, unlike Keap1. Similarly, Nrf2 was shown to be down regulated in hypoxic conditions by the hypoxia-activated E3 ubiquitin ligase Siah2 (seven in absentia homolog 2) [57]. These alternative modes of down regulating Nrf2 could have an important biological significance, especially in situations where Keap1 is functionally inactive.

While this study offers an innovative approach to monitor degradation of Nrf2 in living cells, it is not without limitations. Although we used HepG2 cells stably expressing Dendra2-Nrf2, the expression level of Dendra2-Nrf2 varied between cells presumably due to variability associated with transfection. The heterogeneous expression of Dendra2-Nrf2 in the selected cell population depicted by differences in the initial green fluorescence led to similar differences in the amount of photoconverted red proteins and accounts for the variations in the standard deviations. Additionally, we observed a redistribution of red nuclear Dendra2-Nrf2 throughout the whole cell, which obscures analysis of degradation solely within the nuclear compartment. However, monitoring decay of red fluorescence throughout the whole cell enabled us to measure the absolute half-life of Dendra2-Nrf2 that was initially photoconverted in the nucleus. Despite these caveats, the photoconversion property of Dendra2 coupled with co-expression of Crimson-PML-I provides a novel tool for studying the dynamics of Nrf2 in bona-fide PML-NBs within the nucleus.

Further studies using Dendra2-Nrf2 sumoylation site mutant(s) could help delineate the role of sumoylation-dependent degradation of Nrf2 in the nucleus and PML-NBs. Additional studies measuring the rate of degradation of Dendra2-Nrf2 under conditions of oxidative stress could also uncover time-dependent changes in the abundance of Nrf2. Using this photoconversion paradigm, it is also possible to measure Keap1-dependent degradation in Keap1 null cells by exogenously expressing Keap1, which may be a subject for further investigation. In conclusion, our findings provide direct evidence for degradation of Nrf2 in the nucleus and suggest that modification of Nrf2 in PML nuclear bodies contributes to its degradation in intact cells.

\section{Acknowledgements}

We thank Dr. Peter Hemmerich of Leibniz-Institute of Age Research, Fritz-LipmanInstitute, Jena, Germany for providing us with the expression plasmid for GFP-PML-I; Dr. David 


\section{Cellular Physiology Cell Physiol Biochem 2018;47:800-816 \begin{tabular}{l|l} 
and Biochemistry Published online:May 28, 2018 & $\begin{array}{l}\text { D) } 2018 \text { The Author(s). Published by S. Karger AG, Basel } \\
\text { www.karger.com/cpb }\end{array}$
\end{tabular}}

Burroughs et al.: PML-Nuclear Bodies Regulate the Stability of Nrf2

Piston of Washington University in St. Louis for the original expression plasmid for Dendra2 and Alexander Bläßle of Friedrich Miescher Laboratory of the Max Planck Society, Tübingen, Germany for technical assistance with the PyFDAP software. We thank Dr. Pius Nde, Dr. Smita Misra, Dr. Ujjal Singha at the Department of Immunology and Microbiology, Meharry Medical College for assistance with cloning procedures; members of the Bioinformatics and Morhphology cores at Meharry Medical College for access to these facilities and help with data acquisition and analysis; and the late Dr. Yumiko Kawai for advice during the performance of this work.

This work was supported by NIH grant SC1CA143985, SC1CA211030 and CTSA award No. UL1TR000445 from the National Center for Advancing Translational Sciences. Its contents are solely the responsibility of the authors and do not necessarily represent official views of the National Center for Advancing Translational Sciences or the National Institutes of Health. Confocal microscopy images were generated at the Meharry Medical College Morphology Core Laboratory, which is supported, in part, by NIH grants U54MD007593, G12MD007586, U54CA163069, R24DA036420, and S10RR0254970.

Supported at various times by NIH RISE grant 5R25GM059994 to the School of Graduate Studies and Research, Meharry Medical College and Pharmacology Training grant 5T32GM007628-37 to Vanderbilt University School of Medicine. Supported by NIH grant 4R25GM059994. Dr. Ifeanyi J. Arinze passed away during the preparation of this manuscript

\section{Disclosure Statement}

The authors declare no conflicts of interest.

\section{References}

1 Nguyen T, Sherratt PJ, Pickett CB: Regulatory mechanisms controlling gene expression mediated by the antioxidant response element. Annu Rev Pharmacol Toxicol 2003;43:233-260.

-2 Motohashi H, Yamamoto M: Nrf2-Keap1 defines a physiologically important stress response mechanism. Trends Mol Med 2004;10:549-557.

-3 Wakabayashi N, Dinkova-Kostova AT, Holtzclaw WD, Kang MI, Kobayashi A, Yamamoto M, Kensler TW, Talalay P: Protection against electrophile and oxidant stress by induction of the phase 2 response: fate of cysteines of the Keap1 sensor modified by inducers. Proc Natl Acad Sci U S A 2004;101:2040-2045.

4 Malhotra D, Portales-Casamar E, Singh A, Srivastava S, Arenillas D, Happel C, Shyr C, Wakabayashi N, Kensler TW, Wasserman WW, Biswal S: Global mapping of binding sites for Nrf2 identifies novel targets in cell survival response through ChIP-Seq profiling and network analysis. Nucleic Acids Res 2010;38:57185734.

5 Kensler TW, Wakabayashi N, Biswal S: Cell survival responses to environmental stresses via the Keap1Nrf2-ARE pathway. Annu Rev Pharmacol Toxicol 2007;47:89-116.

6 Hayes JD, Dinkova-Kostova AT: The Nrf2 regulatory network provides an interface between redox and intermediary metabolism. Trends Biochem Sci 2014;39:199-218.

-7 Li J, Lee JM, Johnson JA: Microarray analysis reveals an antioxidant responsive element-driven gene set involved in conferring protection from an oxidative stress-induced apoptosis in IMR-32 cells. J Biol Chem 2002;277:388-394.

8 Kwak MK, Wakabayashi N, Itoh K, Motohashi H, Yamamoto M, Kensler TW: Modulation of gene expression by cancer chemopreventive dithiolethiones through the Keap1-Nrf2 pathway. Identification of novel gene clusters for cell survival. J Biol Chem 2003;278:8135-8145.

-9 Kobayashi A, Kang MI, Okawa H, Ohtsuji M, Zenke Y, Chiba T, Igarashi K, Yamamoto M: Oxidative stress sensor Keap1 functions as an adaptor for Cul3-based E3 ligase to regulate proteasomal degradation of Nrf2. Mol Cell Biol 2004;24:7130-7139.

10 Zhang DD, Lo SC, Cross JV, Templeton DJ, Hannink M: Keap1 is a redox-regulated substrate adaptor protein for a Cul3-dependent ubiquitin ligase complex. Mol Cell Biol 2004;24:10941-10953.

11 Katoh Y, Iida K, Kang MI, Kobayashi A, Mizukami M, Tong KI, McMahon M, Hayes JD, Itoh K, Yamamoto M: Evolutionary conserved N-terminal domain of Nrf2 is essential for the Keap1-mediated degradation of the protein by proteasome. Arch Biochem Biophys 2005;433:342-350. 


\section{Cellular Physiology Cell Physiol Biochem 2018;47:800-816 \begin{tabular}{c|c|c|} 
DOI: 10.1159/000490033 & O 2018 The Author(s). Published by S. Karger AG, Basel \\
wwww.karger.com/cpb
\end{tabular}}

Burroughs et al.: PML-Nuclear Bodies Regulate the Stability of Nrf2

12 McMahon M, Thomas N, Itoh K, Yamamoto M, Hayes JD: Redox-regulated turnover of Nrf2 is determined by at least two separate protein domains, the redox-sensitive Neh2 degron and the redox-insensitive Neh6 degron. J Biol Chem 2004;279:31556-31567.

13 Rada P, Rojo AI, Chowdhry S, McMahon M, Hayes JD, Cuadrado A: SCF/ $\{$ beta\}-TrCP promotes glycogen synthase kinase 3-dependent degradation of the Nrf2 transcription factor in a Keap1-independent manner. Mol Cell Biol 2011;31:1121-1133.

14 Chowdhry S, Zhang Y, McMahon M, Sutherland C, Cuadrado A, Hayes JD: Nrf2 is controlled by two distinct beta-TrCP recognition motifs in its Neh6 domain, one of which can be modulated by GSK-3 activity. Oncogene 2013;32:3765-3781.

15 Nguyen T, Sherratt PJ, Nioi P, Yang CS, Pickett CB: Nrf2 controls constitutive and inducible expression of ARE-driven genes through a dynamic pathway involving nucleocytoplasmic shuttling by Keap1. J Biol Chem 2005;280:32485-32492.

16 Nguyen T, Nioi P, Pickett CB: The Nrf2-antioxidant response element signaling pathway and its activation by oxidative stress. J Biol Chem 2009;284:13291-13295.

17 Kaspar JW, Niture SK, Jaiswal AK: Nrf2:INrf2 (Keap1) signaling in oxidative stress. Free Radic Biol Med 2009;47:1304-1309.

18 Chudakov DM, Lukyanov S, Lukyanov KA: Tracking intracellular protein movements using photoswitchable fluorescent proteins PS-CFP2 and Dendra2. Nat Protoc 2007;2:2024-2032.

19 Zhang L, Gurskaya NG, Merzlyak EM, Staroverov DB, Mudrik NN, Samarkina ON, Vinokurov LM, Lukyanov S, Lukyanov KA: Method for real-time monitoring of protein degradation the single cell level. Biotechniques 2007;42:446, 448, 450.

20 Chudakov DM, Lukyanov S, Lukyanov KA: Using photoactivatable fluorescent protein Dendra2 to track protein movement. Biotechniques 2007;42:553, 555, 557 passim.

21 Chudakov DM, Lukyanov S, Lukyanov KA: Fluorescent proteins as a toolkit for in vivo imaging. Trends Biotechnol 2005;23:605-613.

22 Theodore M, Kawai Y, Yang J, Kleshchenko Y, Reddy SP, Villalta F, Arinze IJ: Multiple nuclear localization signals function in the nuclear import of the transcription factor Nrf2. J Biol Chem 2008;283:8984-8994.

23 Muller P, Rogers KW, Jordan BM, Lee JS, Robson D, Ramanathan S, Schier AF: Differential diffusivity of Nodal and Lefty underlies a reaction-diffusion patterning system. Science 2012;336:721-724.

-24 Rogers KW, Blassle A, Schier AF, Muller P: Measuring protein stability in living zebrafish embryos using fluorescence decay after photoconversion (FDAP). J Vis Exp 2015:52266.

25 Nguyen T, Huang HC, Pickett CB: Transcriptional regulation of the antioxidant response element. Activation by Nrf2 and repression by MafK. J Biol Chem 2000;275:15466-15473.

26 Lin YZ, Yao SY, Veach RA, Torgerson TR, Hawiger J: Inhibition of nuclear translocation of transcription factor NF-kappa B by a synthetic peptide containing a cell membrane-permeable motif and nuclear localization sequence. J Biol Chem 1995;270:14255-14258.

-27 Torgerson TR, Colosia AD, Donahue JP, Lin YZ, Hawiger J: Regulation of NF-kappa B, AP-1, NFAT, and STAT1 nuclear import in T lymphocytes by noninvasive delivery of peptide carrying the nuclear localization sequence of NF-kappa B p50. J Immunol 1998;161:6084-6092.

28 Liu XY, Robinson D, Veach RA, Liu D, Timmons S, Collins RD, Hawiger J: Peptide-directed suppression of a pro-inflammatory cytokine response. J Biol Chem 2000;275:16774-16778.

29 Alvarez-Maqueda M, El Bekay R, Alba G, Monteseirin J, Chacon P, Vega A, Martin-Nieto J, Bedoya FJ, Pintado E, Sobrino F: 15-deoxy-delta 12, 14-prostaglandin J2 induces heme oxygenase-1 gene expression in a reactive oxygen species-dependent manner in human lymphocytes. J Biol Chem 2004;279:21929-21937.

-30 Alam J, Killeen E, Gong P, Naquin R, Hu B, Stewart D, Ingelfinger JR, Nath KA: Heme activates the heme oxygenase-1 gene in renal epithelial cells by stabilizing Nrf2. Am J Physiol Renal Physiol 2003;284:F743-752.

-31 Endo H, Sugioka Y, Nakagi Y, Saijo Y, Yoshida T: A novel role of the NRF2 transcription factor in the regulation of arsenite-mediated keratin 16 gene expression in human keratinocytes. Environ Health Perspect 2008;116:873-879.

32 Li W, Jain MR, Chen C, Yue X, Hebbar V, Zhou R, Kong AN: Nrf2 Possesses a redox-insensitive nuclear export signal overlapping with the leucine zipper motif. J Biol Chem 2005;280:28430-28438.

-33 Li W, Yu SW, Kong AN: Nrf2 possesses a redox-sensitive nuclear exporting signal in the Neh5 transactivation domain. J Biol Chem 2006;281:27251-27263.

34 Malloy MT, McIntosh DJ, Walters TS, Flores A, Goodwin JS, Arinze IJ: Trafficking of the transcription factor Nrf2 to promyelocytic leukemia-nuclear bodies: implications for degradation of NRF2 in the nucleus. J Biol Chem 2013;288:14569-14583. 


\section{Cellular Physiology Cell Physiol Biochem 2018;47:800-816

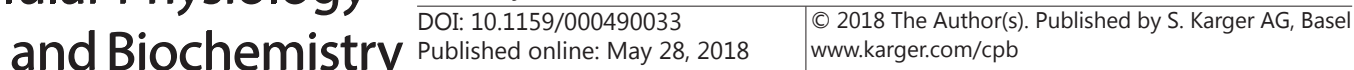

Burroughs et al.: PML-Nuclear Bodies Regulate the Stability of Nrf2

35 Ben-Dor A, Steiner M, Gheber L, Danilenko M, Dubi N, Linnewiel K, Zick A, Sharoni Y, Levy J: Carotenoids activate the antioxidant response element transcription system. Mol Cancer Ther 2005;4:177-186.

-36 Lallemand-Breitenbach V, de The H: PML nuclear bodies. Cold Spring Harb Perspect Biol 2010;2:a000661.

-37 Condemine W, Takahashi Y, Zhu J, Puvion-Dutilleul F, Guegan S, Janin A, de The H: Characterization of endogenous human promyelocytic leukemia isoforms. Cancer Res 2006;66:6192-6198.

-38 Dellaire G, Bazett-Jones DP: PML nuclear bodies: dynamic sensors of DNA damage and cellular stress. Bioessays 2004;26:963-977.

39 Bernardi R, Pandolfi PP: Structure, dynamics and functions of promyelocytic leukaemia nuclear bodies. Nat Rev Mol Cell Biol 2007;8:1006-1016.

-40 Eskiw CH, Dellaire G, Mymryk JS, Bazett-Jones DP: Size, position and dynamic behavior of PML nuclear bodies following cell stress as a paradigm for supramolecular trafficking and assembly. J Cell Sci 2003;116:4455-4466.

41 Kamitani T, Kito K, Nguyen HP, Wada H, Fukuda-Kamitani T, Yeh ET: Identification of three major sentrinization sites in PML. J Biol Chem 1998;273:26675-26682.

-42 Sahin U, Ferhi O, Jeanne M, Benhenda S, Berthier C, Jollivet F, Niwa-Kawakita M, Faklaris O, Setterblad N, de The H, Lallemand-Breitenbach V: Oxidative stress-induced assembly of PML nuclear bodies controls sumoylation of partner proteins. J Cell Biol 2014;204:931-945.

43 Hayes JD, McMahon M: NRF2 and KEAP1 mutations: permanent activation of an adaptive response in cancer. Trends Biochem Sci 2009;34:176-188.

44 Ashino T, Ohkubo-Morita H, Yamamoto M, Yoshida T, Numazawa S: Possible involvement of nuclear factor erythroid 2-related factor 2 in the gene expression of Cyp2b10 and Cyp2a5. Redox Biol 2014;2:284-288.

45 Nguyen T, Sherratt PJ, Huang HC, Yang CS, Pickett CB: Increased protein stability as a mechanism that enhances Nrf2-mediated transcriptional activation of the antioxidant response element. Degradation of Nrf2 by the 26 S proteasome. J Biol Chem 2003;278:4536-4541.

-46 Reddy NM, Kleeberger SR, Yamamoto M, Kensler TW, Scollick C, Biswal S, Reddy SP: Genetic dissection of the Nrf2-dependent redox signaling-regulated transcriptional programs of cell proliferation and cytoprotection. Physiol Genomics 2007;32:74-81.

47 Suzuki T, Motohashi H, Yamamoto M: Toward clinical application of the Keap1-Nrf2 pathway. Trends Pharmacol Sci 2013;34:340-346.

48 Thimmulappa RK, Mai KH, Srisuma S, Kensler TW, Yamamoto M, Biswal S: Identification of Nrf2-regulated genes induced by the chemopreventive agent sulforaphane by oligonucleotide microarray. Cancer Res 2002;62:5196-5203.

49 Singh A, Misra V, Thimmulappa RK, Lee H, Ames S, Hoque MO, Herman JG, Baylin SB, Sidransky D, Gabrielson E, Brock MV, Biswal S: Dysfunctional KEAP1-NRF2 interaction in non-small-cell lung cancer. PLoS Med 2006;3:e420.

50 Ohta T, Ijijima K, Miyamoto M, Nakahara I, Tanaka H, Ohtsuji M, Suzuki T, Kobayashi A, Yokota J, Sakiyama T, Shibata T, Yamamoto M, Hirohashi S: Loss of Keap1 function activates Nrf2 and provides advantages for lung cancer cell growth. Cancer Res 2008;68:1303-1309.

-51 Konstantinopoulos PA, Spentzos D, Fountzilas E, Francoeur N, Sanisetty S, Grammatikos AP, Hecht JL, Cannistra SA: Keap1 mutations and Nrf2 pathway activation in epithelial ovarian cancer. Cancer Res 2011;71:5081-5089.

52 Yoo NJ, Kim HR, Kim YR, An CH, Lee SH: Somatic mutations of the KEAP1 gene in common solid cancers. Histopathology 2012;60:943-952.

53 Guo S, Cheng X, Lim JH, Liu Y, Kao HY: Control of antioxidative response by the tumor suppressor protein PML through regulating Nrf2 activity. Mol Biol Cell 2014;25:2485-2498.

54 Niwa-Kawakita M, Ferhi O, Soilihi H, Le Bras M, Lallemand-Breitenbach V, de The H: PML is a ROS sensor activating p53 upon oxidative stress. J Exp Med 2017

55 Rada P, Rojo AI, Evrard-Todeschi N, Innamorato NG, Cotte A, Jaworski T, Tobon-Velasco JC, Devijver H, Garcia-Mayoral MF, Van Leuven F, Hayes JD, Bertho G, Cuadrado A: Structural and functional characterization of Nrf2 degradation by the glycogen synthase kinase 3/beta-TrCP axis. Mol Cell Biol 2012;32:3486-3499.

-56 Kang HJ, Hong YB, Kim HJ, Bae I: CR6-interacting factor 1 (CRIF1) regulates NF-E2-related factor 2 (NRF2) protein stability by proteasome-mediated degradation. J Biol Chem 2010;285:21258-21268.

57 Baba K, Morimoto H, Imaoka S: Seven in absentia homolog 2 (Siah2) protein is a regulator of NF-E2-related factor 2 (Nrf2). J Biol Chem 2013;288:18393-18405. 